\title{
A multi-objective decision-based solution (MODM) for facility location-allocation problem using cuckoo search and genetic algorithms
}

\author{
Amir Shimi $^{1}$. Mohammad Reza Ebrahimi Dishabi ${ }^{1}$. Mohammad Abdollahi Azgomi
}

\begin{abstract}
Considering the significant advancements in autonomous vehicle technology, research in this field is of interest to researchers. To automatic parking, controlling steer angle, gas hatch, and brakes need to be learned. Due to the increase in the number of cars and road traffic, car parking space has decreased. Its main reason is information error. Because the driver does not receive the necessary information or receives it too late, he cannot take appropriate action against it. This paper uses two phases: the first phase, for goal coordination, was used genetic algorithms and the Cuckoo search algorithm was used to increase driver information from the surroundings. Using the Cuckoo search algorithm and considering the limitations, it increases the driver's level of information from the environment. Also, by exchanging information through the application, it enables the information to reach the driver much more quickly and the driver reacts appropriately at the right time. The suggested protocol is called the MODM-based solution. Here, the technique is assessed through extensive simulations performed in the NS-3 environment. Based on the simulation outcomes, it is indicated that the parking system performance metrics are enhanced based on the detection rate, false-negative rate, and false-positive rate.
\end{abstract}

Keywords Location allocation problem. Cuckoo search algorithm. Automatic parking. MODMbased solution.

Amir Shimi

Shimi@m-iau.ac.ir

Mohammad Reza Ebrahimi Dishabi

mrebrahimy@m-iau.ac.ir

$\checkmark$ Mohammad Abdollahi Azgomi

Azgomi@iust.ac.ir

${ }^{1}$ Department of Computer Engineering Miyaneh Branch, Islamic Azad University, Miyaneh, Iran

${ }^{2}$ School of Computer Engineering, Iran University of Science and Technology, Hengam St., Resalat Sq., Tehran, Iran 


\section{Introduction}

In the location problem, we address allocating facilities while considering the multi-objective function in line with optimization of the target function such that candidate locations for deploying facilities are specified. A number of facility applicants are stationed in specified locations, which are ready to receive services from the facilities. Considering the number of applicants, the capacity of each facility, location where the applicant is stationed, and how the applicant is assigned facilities are among the goals of the multi-objective location-allocation problem [1-3]. Establishing facilities at candidate points leads to expenses in the transportation system, in which the fixed deployment cost and the transportation cost must be minimized. Such problems usually have lots of local optimum points. As a result, accurate algorithms are not usually appropriate methods for solving location-allocation problems. Therefore, to escape from local optimums and generate suitable solutions, meta-heuristic algorithms like the Cuckoo algorithm, which have presented good results in solving such problems, can be considered. Figure 1 shows the autonomous driving system in this research.

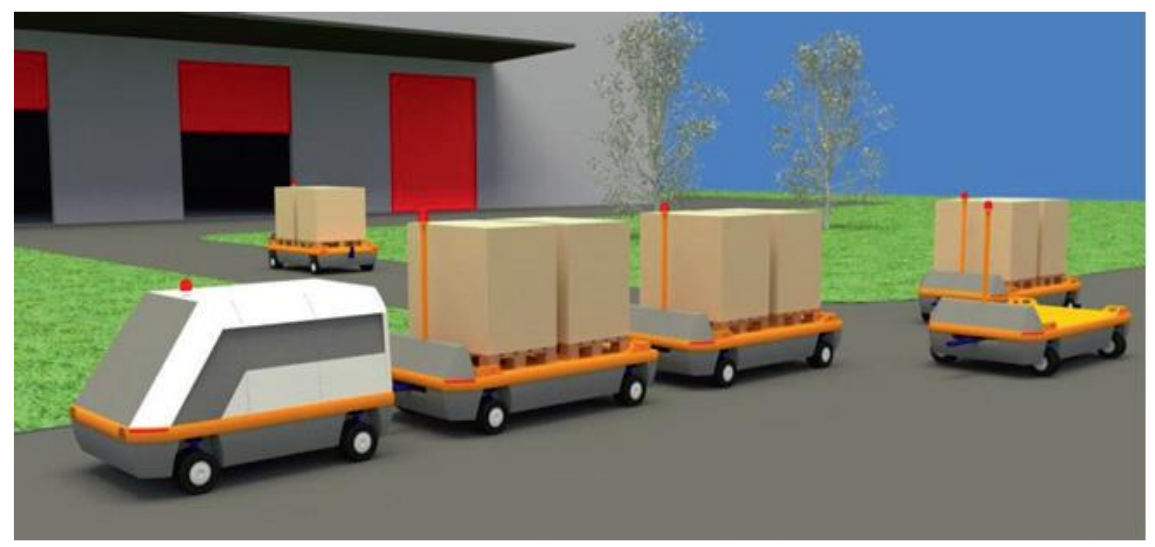

Fig. 1 Automatic parking vehicle [2].

To mathematically model the multi-objective location problem with the goal of minimizing cost and maximizing applicant absorption, a network is considered where the nodes represent the deployment location and the relationships between them are the possible routes [4-6]. We solve the problem by taking parameters like quality, time, and cost of selecting facilities with higher absorption rates into consideration. Due to the randomness of applicant demands and limitation of capacity, we will carry out the decision-making of the problem using meta-heuristic algorithms. The main objective is to select the optimal facility point to minimize the target function. By considering the set of demand points as follows. Set of demonstrate points is demonstrate in Table 1.

Table 1: Set of demand points.

\begin{tabular}{|l|l|}
\hline Parameters & Value \\
\hline $\mathrm{R}$ & $\{1,2,3, \ldots\}$ \\
\hline And demand with parameter i & \\
\hline Mean & $M$ \\
\hline Variance & $V$ \\
\hline Location set & $H=\{1,2,3, \ldots\}$ \\
\hline Facility capacity with parameter j & \\
\hline Level of service & $A$ \\
\hline Quality & $Q$ \\
\hline
\end{tabular}


To deal with the applicant's uncertainty, we follow the normal distribution and mean and variance. Considering the uncertainty in customer demand, in this study the customer demand is met based on the capacity constraint. For mathematical modeling, the multi-objective locating problem is considered in which the nodes represent the location and the relationship between the nodes are possible ways to minimize the cost and maximize the applicant attraction through the network. The three factors of service, quality and travel cost are used to express customer preferences. The cost of receiving service inversely influences the customer choice. For each facility, a specific level of service is considered. We solve the problem considering the parameters such as quality, time and the cost of selecting facilities with higher attraction. Due to the randomness of applicant requests and capacity constraints, we will make decision about the problem using heuristic algorithms. The main objective is to select the optimal location for the facility to minimize the objective function. Considering a set of demand points, we select a collection called $\mathrm{R}$ with the demands as members and a parameter $\mathrm{i}$ as the counter of demands. Here, regarding the randomness of the requests, random parameters of variance and standard deviation is applied to calculate the measure of variability and the nearer and farther demands. Here, $\mathrm{V}\left(\sigma^{2}\right)$ and $\sigma$ represent variance and standard deviation respectively. In this formula:

Therefore, the limitation is defined in Eq. (1):

$$
P\left(\frac{\sum_{i=1}^{n} a_{i} x_{i j}-\sum_{i=1}^{n} \mu_{i} x_{i j}}{\sqrt{\sum_{i=1}^{n} \sigma_{i}^{2} x_{i j}^{2}}} \leq \frac{L_{j} y_{j}-\sum_{i=1}^{n} \mu_{i} x_{i j}}{\sqrt{\sum_{i=1}^{n} \sigma_{i}^{2} x_{i j}^{2}}}\right) \geq \alpha_{j} \forall_{j} \in J
$$

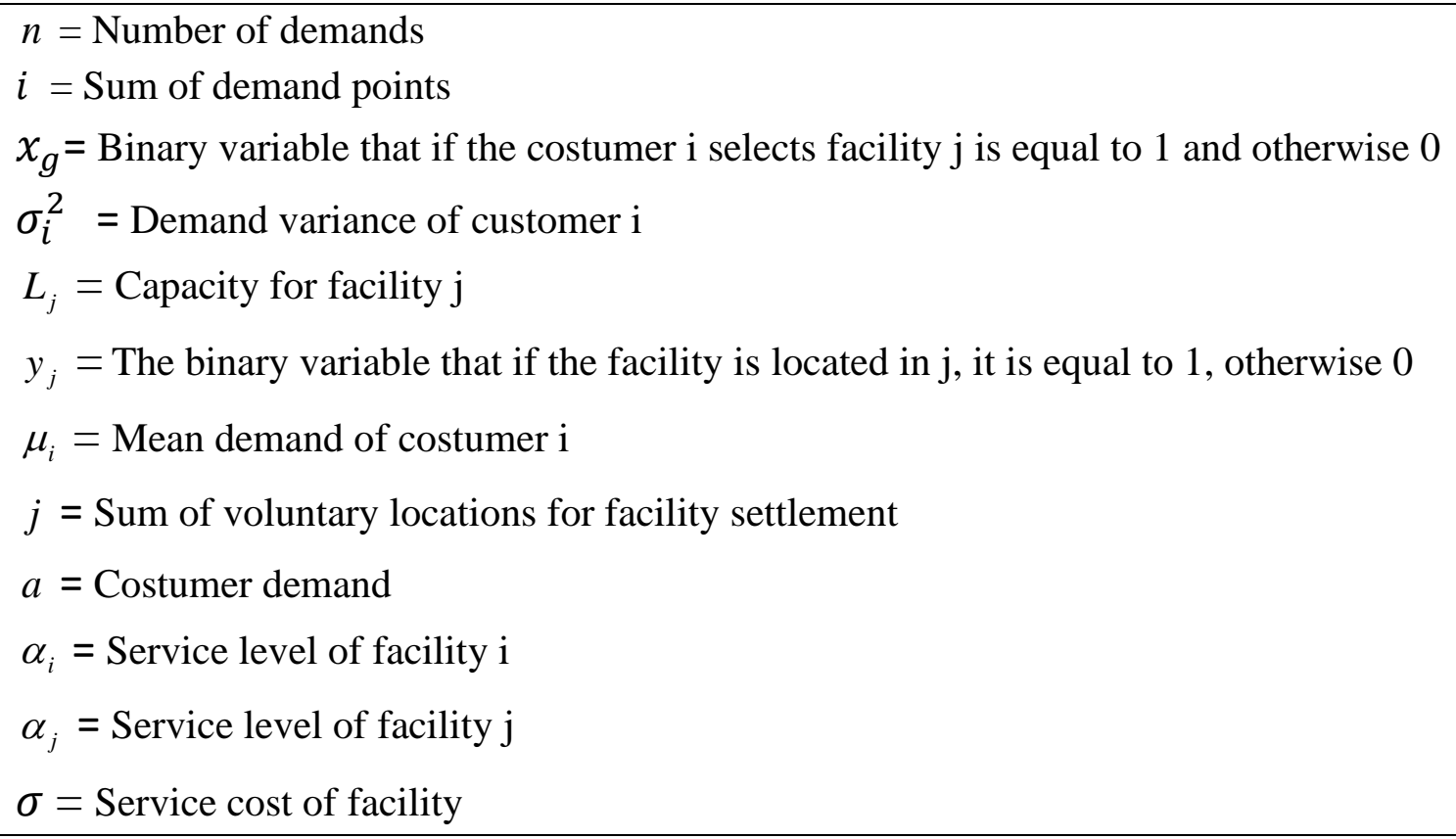

In the formula above, the normal distribution of variance and mean is followed to control the uncertainty of customer demands and we convert the probability constraint to obsolete constraint. 
Since the customer demand is independent and has normal distribution, the demand for facility $\mathrm{j}$ also follows the normal distribution.

Therefore, the limitation is as follows: in this study, which seeks to locate parking, for instance we can try to specify the optimal and suitable locations for building the parking by applying rules and criteria like distance from population absorbent centres, distance from passages, and suitable usages for constructing a parking and enforcing limitations and unsuitable usages for constructing a parking. To this end, first, the criteria effective in locating Multi Storey car parks are weighted using the hierarchical analysis process and the best location for construction is chosen with respect to the resulting weights. To solve this optimization problem, it is essential for problem variables to be formed as an array. For example, in the genetic algorithm and the particle algorithm, these arrays are specified with "chromosome" and "particle position" names. However, in the Cuckoo optimization algorithm, this array is called "habitat". In an N-dimensional optimization problem, a habitat will be an $1 * N$ array which represents the current living location of cuckoos [7]. This array is defined as follows Eq. (2):

$$
\text { Habitat }=\left[x_{1}, x_{2}, x_{3}, \ldots, x_{N}\right]
$$

The appropriateness (or amount of profit) in the current habitat is obtained by evaluating the profit function (fp) in the habitat. Therefore in Eq. (3):

$$
\text { Profit }=\mathrm{fp}(\text { habitat })=f p\left(x_{1}, x_{2}, x_{3}, \ldots, x_{N}\right)
$$

As can be seen, the evolutionary cuckoo algorithm is an algorithm that maximizes the profit function. To use the cuckoo algorithm for solving minimization problems it is enough to multiply a negative sign in the cost function. To start the optimization algorithm, a habitat matrix of size $N_{p o p} * N$ is created. Then, a number of eggs are assigned to each one of the habitats. In nature, each cuckoo lay between 5 and 20 eggs. These numbers are used as the higher and lower bounds of assigning eggs to each cuckoo in different iterations. Another habit of a real cuckoo is that they lay their eggs in a specific domain which is called the Egg Laying Radius (ELR) [8]. In an optimization problem, each variable has an upper limit $\left(v a r_{\text {hi }}\right)$ and lower limit $\left(\right.$ var $\left._{\text {low }}\right)$ where each ELR will be definable using these limits. ELR is proportionate to the total number of eggs, number of cuckoo's current eggs, and also the upper and lower limits of the problem. Therefore, ELR is calculated as Eq. (4).

$$
E L R=\alpha *\left(\frac{\text { Number of cuurent cuckoo seggs }}{\text { Total number of eggs }} *\left(\operatorname{Var}_{h i}-\operatorname{Var}_{\text {low }}\right)\right)
$$

For solving the location problem, one could pick an arbitrary vertex with minimum degree and call it v. Create the layer structure $L_{v}$ with root v. We get $\mathrm{S}$ to be the set of vertices at the last layer of $L_{v}$ (vertices that have the maximum distance from vertex $v$ ). We create these vertices in order of increasing degree. Imagine $u$ is a vertex of $S$, the layer structure of which has the lowest width. The algorithm terminates with $\mathrm{u}$ and $\mathrm{v}$ as terminal points. To minimize, we convert the two-layer structures created in the last step to a new layer structure whose width is less than both the structures. To do this, 
swarm optimization algorithms for route optimization can be used and obtain satisfactory results in multi-objective optimization algorithms by considering multiple objectives for the optimization of the target location function.

This paper uses two phases: the first phase, for goal coordination, was used genetic algorithms and the Cuckoo search algorithm was used to increase driver information from the surroundings. Using the Cuckoo search algorithm and considering the limitations, it increases the driver's level of information from the environment. Also, by exchanging information through the application, it enables the information to reach the driver much more quickly and the driver reacts appropriately at the right time.

The presented work is structured as the following. Section 2 presents cuckoo search algorithm and related works. In Sect. 3 brings the proposed MODM-Based solution. The parameters used for assessing the performance are studied and simulation outcomes are deliberated in Section 4. Finally, conclusion of this research is discussed in Section 5.

\section{Preliminaries}

In this section, we provide a brief review over some basic issues such as the cuckoo search algorithm and related works.

\subsection{Cuckoo Search Algorithm}

Cuckoo Search (CS), which is one of the recent search algorithms inspired by nature, was developed by Suash Deb of C.V. Raman College of Engineering and Xin-She Yang of Cambridge University in 2009. This algorithm is on the basis of the brood parasitism of cuckoo types. Deb and Yang presented three ideals for application of CS as an optimization means [9]:

- One egg is laid by each cuckoo at a time, and it discharges the egg in a nest that is randomly selected;

- The nests with the high-quality eggs are transferred to the subsequent generations;

- There is a fixed number of present host nests, and a host bird detects the egg laid by a cuckoo with $\mathrm{Pa} \in[0.1]$ probability.

In this way, the host bird has two choices: getting rid of the egg, or just abandoning the nest and constructing a totally new nest. As a more approximation, this assumption would be approximated by a fraction $\mathrm{Pa}$ of the $\mathrm{N}$ host nests that are substituted by new ones (new random solutions). For the implementation purpose, new solutions (cuckoos) can be generated by two ways: Le'vy flight and a fraction $\mathrm{Pa}$ of the $\mathrm{n}$ host nests substituted by new random solutions. First, Le'vy flight is considered. It was utilized already in search algorithms. It is a random walk described by some instant bounces selected from a probability density function with a power-law tail. When we generate new solutions $\mathrm{x}(\mathrm{t}+1)$ for a cuckoo called $\mathrm{i}$, the following equation is used for performing a Le'vy flight:

$$
x_{i}^{(t+1)}=x_{i}^{(t)}+\alpha \oplus L e^{\prime} v y(\psi)
$$

where a $>0$ denotes the size of the step that must be associated with the respective problem scale. Most of the time, $\mathrm{a}=(\mathrm{L} / 10)$ where $\mathrm{L}$ denotes the distinctive scale of the respective problem. Equation (5) is the stochastic one that is used for a random walk. Generally, a random walk is defined as a 
Markov chain that its subsequent location/status is just dependent on the current location (the $1^{\text {st }}$ term of Eq. 5) and the probability of the transition (the $2^{\text {nd }}$ term of Eq. 5). The meaning of the product $\oplus$ is entry-wise multiplications. It is like the products that are utilized in PSO. However, the random walk by Le'vy flight here has higher efficiency to explore the search space since it has a much longer step length in the long-term [10]. The random walk's step length developed by Le'vy flight follows the Le'vy distribution that has an infinite mean and variance as in Equation (6):

$$
L e^{\prime} v y \sim u=t_{i}^{-\psi *} *(1 \prec \psi \leq 3)
$$

where $\psi$ represents a constant, and it is possible to mathematically calculate the step length by the Equation (7):

$$
L e^{\prime} v y(\psi)=\left(\frac{u}{|v|^{\frac{1}{\psi}}}\right)
$$

where $\mathrm{v}$ and $\mathrm{u}$ are taken from normal distributions as follows:

$$
u \sim N\left(0 * \partial_{u}^{2}\right) * v \sim N\left(0 * \partial_{v}^{2}\right)
$$

where:

$$
\partial_{u}=\left\{\left(\frac{r(1+\psi) \sin \left(\frac{\pi \psi}{2}\right)}{r\left[\frac{1+\psi}{2}\right] \psi 2 \frac{(\psi-1)}{2}}\right)^{\frac{1}{\psi}} . \partial_{v}=1\right.
$$

Shortly, the step length substantially creates a random walk with a heavy tail and a power-law steplength distribution. Some of the novel's solutions need to be created by Le'vy walk around the best solution gained up to now. Hence, this accelerates the local search. Additionally, new random solutions replace a fraction $\mathrm{Pa}$ of the solutions, and their locations must be adequately far from the present best solution. Thus, it would be assured that the system would not be caught by a local optimum.

\subsection{Related work}

Cyprus International Airport decided to use the Hikvision smart parking system to minimize the problems caused by parking cars and protecting them. They left the access control system, the smart airport parking system, and also monitoring other parts of the airport to the closed-circuit camera system of Hikvision. In this solution of Hikvision, the barriers installed in the car park entrance are controlled smartly using sensors and special radar. Efficiency of this model has other advantages in addition to easy installation and setup which include not being affected by environmental factors like lighting, dust, 
and rain. In addition to the automatic and smart barrier control capability, this system can leave some of the control manually to the operator. By using smart algorithms and modern technologies in the security and closed-circuit field, the Hikvision smart system can present valuable statistical and analytical information to the managers so that, if necessary, they can make important decisions using this information and increase security and customer satisfaction levels [12].

In some studies that Zung et al. conducted for understanding the decisions in car parks by modeling the structural equation, they have created a structural equation for analyzing parking decisions. The data used in this study was obtained from the Information Park in Beijing. The relationships between three parking decisions were studied. The results show a two-way correlation between street park and duration. These findings can be used for developing some measures for regulating ractAnalysis of parking in the car park and the parking mechanism for balancing parking time-time distribution and also formulating parking management policies [13].

Maravel et al. have solved the problem of automatic parking using a rear-wheel drive vehicle using a biometric model based on the direct connection between the perception of the vehicle and actions. This problem has been inspired using the external approach where the vehicle controller does not need to know the car cinématique and dynamics. Also, it does not require previous knowledge of the environment's map. The main point in the proposed approach is the definition of performance indicators that happen to automatic parking and actions are injected to the car robot controller in real time. This solution is in the form of a multi-objective dynamic optimization problem and is extremely analytical. Using the genetic algorithm, they have obtained a very simple and effective solution [14].

In the studies Zhao et al. have conducted on analyzing the activity-based trip chain in the parking fee network program, they incorporated the chaining behavior of activity-based trip in analyzing network stability and an integrated model has been presented for describing the passenger's behavior, which is a combination of Beckman link congestion terms and type two logit demand function. The convexity conditions and equivalence of the model have been discussed. Based on the integrated model, a twolayer model has been designed for maximizing social welfare through suitable parking cost. Also, an extensive network for eliminating services and trips in the main network has been developed. Then, the Simulated annealing (SA) method has been used for solving the proposed two-layer model. Numerical examples have been presented for studying the availability of the model and the effects of parking fee scheme on passengers' behavior and social welfare, which indicate that this model is effective in describing the trip chaining behavior in the network [15].

Courhorn et al. have also presented a method and system for providing information based on the location of user's device. A method and a system for providing information based on the location of a user's device, where user's device can connect to a server. This method consists of identification of the initial location of user's device, creation of an initial geofence around the initial location, retrieval of the initial location from the initial geofence, where the first user's device sends the location once from the first geofence, presenting the initial information set to the user's device based on its initial location, determining change at the location of user's device from the first location to the second location, second location which is located outside the initial geofence, while the second geofence is created around the second location. Then, in the second device, location from the second geofence, which has achieved significant results, provide the second information set to user's device based on the second location [16].

In the bus driving schedule optimization under park and ride in tourism attractions study, Zhang et al. proposed a scheduling optimization method for bus driving in park and ride in tourism attractions. The optimization method can provide high-quality tourism experience by decreasing the passengers' waiting time. The optimization method comprises two main steps. First, modal decomposition is 
proposed for drive tourists based on a logit function. Then, bus driving schedule is designed while considering the dynamic passenger demand based on time-development network of city buses. Numerical results show that the bus driving schedule optimization method can effectively decrease passenger waiting time and can be used for more real tourism attractions [17].

To solve the traffic pressure caused by container trucks in ports, Chang et al. proposed an underground container logistics service (UCLS) between Shanghai terminal and north west of logistics park. To ensure the connection between the system and the terminal, designing an underground parking is proposed. Underground parking is a buffer that is used for loading and unloading underground vehicles (UGVs). A nonlinear ordinary planning model (MNIP) has been designed for UGVs and outdoor cranes in order to minimize the overall cost of the cranes in line for UGVs and terminals. Then, the optimization model has been implemented using MATLAB software [18].

Oren Steen et al. have designed a parking management system that comprises a central dataset in communication with the server, at least one user device, at least one merchant console, and one parking control device in a network. The central database is presented for receiving and storing data from several parking systems. A processor has been presented for analyzing the information received by the central data base. A dynamic data engine has been presented for analyzing the data received from a number of parking systems and generating dynamic data. Targeted advertising engine has been presented for analyzing user's data and creating a targeted advertisement. Dynamic pricing information are given to user's device so that the user can reserve the parking space from one of the parking systems. Targeted promotion is given to user's device so that the user can choose an advertisement from a merchant [19].

Works carried out in Iran related to smart car parks:

a) designing a smart parking management and guidance system and its role in securing and increasing road capacity according to paper [14]. To obtain information, first they have referred to the reality of the society and through interviews, questionnaires, and case studies addressed the issues and problems present regarding car parks. This paper is conducted using the descriptive method and on 70 citizens (driver). In the following, we address some of these questions and tables.

According to Table 2, the results of this study regarding the satisfaction level of drivers from parking services around the city were at the low and medium level and only 6 percent have high satisfaction.

Table 2: Satisfaction level with services [14].

\begin{tabular}{|c|c|c|c|}
\hline Low & Medium & High & Overall \\
\hline 27 & 39 & 4 & 70 \\
\hline $38 \%$ & $56 \%$ & $6 \%$ & $100 \%$ \\
\hline
\end{tabular}

According to Table 3, it shows that locating a parking location by asking the people of the region is the highest at 48 percent. Drivers trying to find a parking spot on the street is second with 30 percent and routing systems with 3 percent has had the lowest usage by drivers.

Table 3: Results of asking people [14].

\begin{tabular}{|c|c|c|c|c|c|}
\hline $\begin{array}{c}\text { Asking } \\
\text { people of the } \\
\text { region }\end{array}$ & $\begin{array}{c}\text { Using } \\
\text { locators }\end{array}$ & Using traffic signs & $\begin{array}{c}\text { Using city } \\
\text { map }\end{array}$ & $\begin{array}{c}\text { None, try to find a } \\
\text { spot on the street }\end{array}$ & Overall \\
\hline 34 & 2 & 9 & 4 & 21 & 70 \\
\hline $48 \%$ & $3 \%$ & $13 \%$ & $6 \%$ & $30 \%$ & $100 \%$ \\
\hline
\end{tabular}


According to the results of Table 4, this question has been raised that how important the factors affecting a parking spot are and therefore, the drivers were asked to score the factors mentioned in the questionnaire according to their effect on choosing a parking spot from one to ten. These results show that some parameters affecting parking selection play a more important role while other factors are less important.

Table 4: Importance weight of different factors effective in ranking

\begin{tabular}{|c|c|}
\hline Parameter effective in ranking & Importance weight \\
\hline Access time to parking & 0.42 \\
\hline Geographic distance & 0.45 \\
\hline Parking fee & 0.51 \\
\hline Security and safety & 0.32 \\
\hline Parking difficulty & 0.12 \\
\hline Predicting the future regarding vehicle exit & 0.24 \\
\hline
\end{tabular}

And in the final question where drivers were asked about highest services to the customers, they ranked mentioned factors from one to four and the results are presented in Table 5. which shows the importance of providing better parking services with regard to market work with the highest share at 38 percent.

Table 5: Maximum services provided to the customers by car parks

\begin{tabular}{|c|c|}
\hline Parameter & Importance weight \\
\hline Dith respect to market work & 0.38 \\
\hline With respect to recreational, cultural, and tourism centers & 0.32 \\
\hline During Norouz, eves, religious festivals, and holidays & 0.17 \\
\hline
\end{tabular}

\section{Works carried out abroad related to smart car parks:}

a) Parking Guidance and Information System (PGIS): As a part of smart transportation systems, it can be useful for solving traffic problems. The state of traffic in big city areas has become severe due to looking for parking spots. Advanced parking guidance and information system is considered one of the most effective traffic management approaches which can control and effect the usage of vehicles, especially in crowded and busy parts of the city. Beijing was chosen to host the 2008 Olympics and the government of China has acknowledged that these games would be the most splendid in Olympics history. Before holding these games, it was evaluated in an overall analysis that one of the factors that would affect Olympic games was the traffic system and Beijing officials decided to provide a comfortable, safe, and accurate traffic system to reduce the negative effects of this matter on Olympic games [20]. Despite the fact that nowadays many traffic indicators of china like road network congestion, number of licensed cars, and number of daily passengers are at the top of global rankings, infrastructure facilities of Beijing are behind the global trend with regards to traffic management.

Traffic properties of Olympics are defined as follows:

- Short-term events

- High congestion

- High demand

One of the tools used by Beijing officials in managing Olympics traffic was controlling and scheduling the traffic in Olympics village. From 1997, Chinese Academy of Sciences has started two massive projects in order to research and develop regarding traffic issues. The initial name of this project was 
"Urban Traffic Flow Guidance System”. Jilin University was responsible for carrying out one of these projects. Due to this reason, a research group was formed to design and build a system for the information and management of car parks. This system was named "Urban Traffic Flow Guidance System" [21]. The scheme for integrating parking guidance with traffic flow guidance in smart transportation systems is shown in Figure 2.

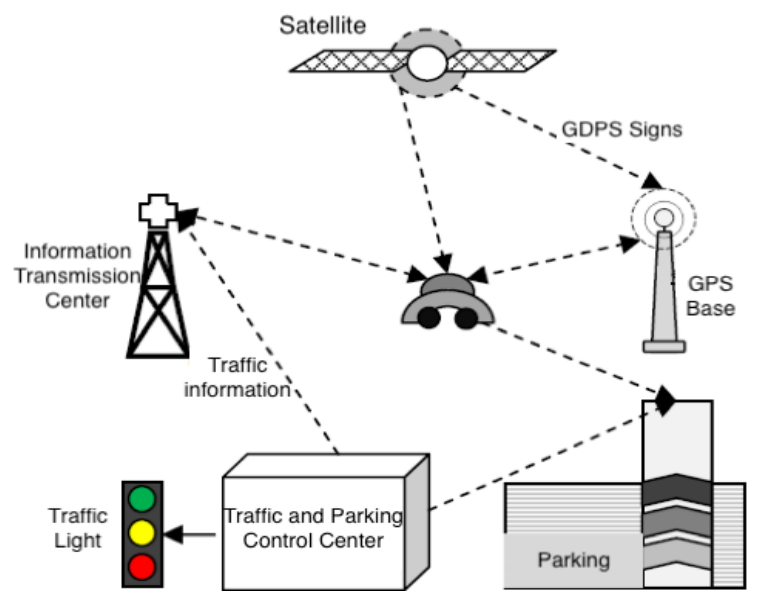

Fig. 2 The scheme for integrating parking guidance with traffic flow guidance in smart transportation systems [21].

Comparison of targeted car parking is shown in Figure 3.
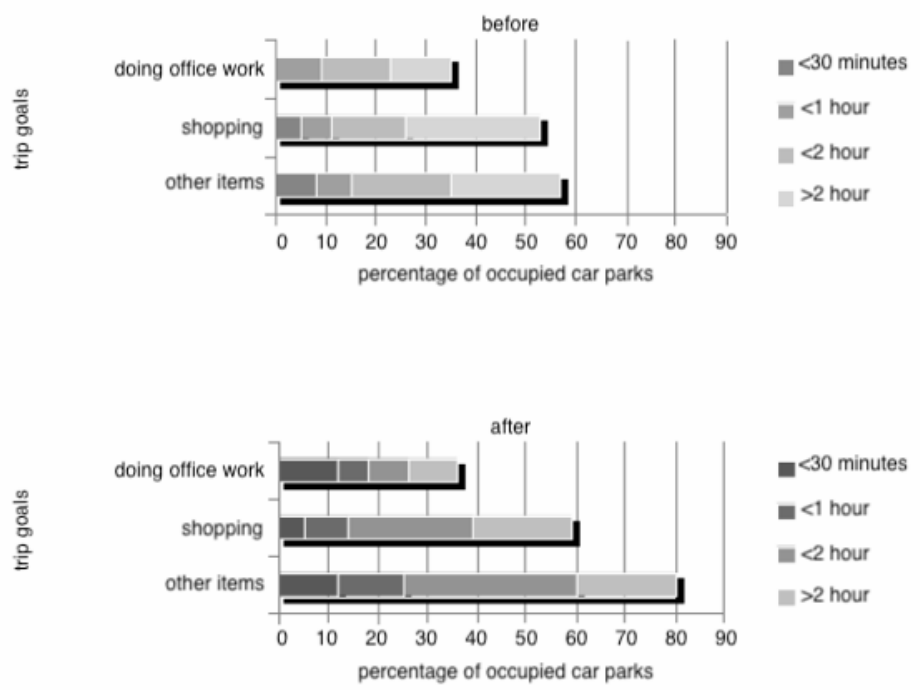

Fig. 3 Comparison of targeted car parking duration before and after utilizing the PGIS system in on of Beijing's regions [21].

b) Smart Driver Location System for Smart Parking: Often, finding a parking spot is tiresome for the drivers and the car park itself is expensive in all the major cities of the world. In this paper, a crowdsourced solution has been proposed that gathers the real information of available car parks using the sensors in smartphones. This system is designed based on cell phones which can follow the driver's route until he or she wants to leave the car park. In this paper, it has been focused on the efficiency and accuracy of using mobile phones for depicting the driver's walking route, which is carried out using the pedestrian dead reckoning (PDR) method installed on the belly and can measure the driver's moving distance with high accuracy. Also, an algorithm synchronous with the map has been designed to measure 
route errors while the driver is indoors (interior environment). It has carried out this deed by utilizing existing floor maps of buildings. The results have shown that it can guess user's walking distance with an approximate accuracy of 98 percent, which along with location errors is about 0.48 meters. In this paper, it has been focused on how to detect the exiting car park activity. This idea is very simple because if the phone detects that the driver is approaching where his or her car is parked, it seems like the driver wants to leave the spot and the parking spot will be available soon.

In this architecture, a driver, who has parked recently, can provide a message regarding when he or she wants to leave the spot and this information might be sold to another driver who wants to pay (using virtual money, like BitCoin). Once the buyer reaches the parking spot when it is close to the departure time of the seller, he can occupy the spot after the seller leaves. Therefore, drivers only exchange the information regarding available car parks. This action is carried out automatically in this paper, i.e. the act of registering and removing parking spots is done automatically. The main focus of this paper is on how the walking path of the driver, which is the key to this method, can be efficiently and accurately depicted. According to this paper [22] and all their previous research, previous systems all worked manually and could not automatically carry out the park registration and removal operation and so the users needed to apply this operation manually. In order for his system to be able to carry out this operation automatically, it needs to monitor user's behaviour.

In [23] two-step optimization process is implemented. Firstly, optimal location and charge-discharge schedule of two PHEVs parking is executed in three different penetration levels by PSO (Particle Swarm Optimization), secondly optimal location and sizing of two SVC is specified by CSA (Cuckoo Search Algorithm) optimization method.

\section{The proposed MODM-Based solution}

We provide a proposed MODM-Based solution in the following section using the genetic algorithms and cuckoo search algorithms. Three phases are included in the MODM-Based solution: in Sect, 3.1. Overview of the MODM-Based solution is discussed, Sect 3.2. presents the Goal coordination with genetic algorithms, and in Sect. 3.3 Cuckoo search algorithm is discussed.

\subsection{Phase 1: Overview of the MODM-Based solution}

The objective of this study is to find a parking management framework which can show the cars moving in and out of the parking and calculate the available parking space and finally, release this information for the customers of the intended parking. For displaying the cars, a magnetic sensor network is used which can collect and estimate the number of cars and also their length and speed while moving in front of the sensors. Also, this information is stored in the data base and when the customer wants to enter the parking, this information is displayed to him or her.

\subsection{Phase 2: Goal coordination with genetic algorithms}

Look at the way a human driver parks the car automatically to observe if any signs could be found for the way of defining proper variables and parameters so that a genetic algorithm can present a solution for the coordination problem. In short, it is observed that parking operation follow these two primary rules [24]: 
1. When the location of the vehicle is far from the target, the approach goal must have priority (index $J_{1}$ ).

2. When the location of the vehicle is near to the target, the heading goal must have priority (index $\left.J_{2}\right)$.

3.

$$
Q(t)=W_{1}(t) Q^{1}(t)+W_{2}(t) Q^{2}(t)
$$

It should be noted that the terms near and far are ambiguous. If the simplest case is considered for linear coordination, then these primary rules of parking goal coordination can be defined in a simple way, which is similar to the standard membership functions employed in the fuzzy subset theory. In this graph, $\rho$ represents the distance of the vehicle from the parking space or target, the interpretation of which is simple. Assume that the coefficients' curves are symmetric, then $\rho_{m}$ and $\rho_{M}$ are the parameters determining the coordination dynamics. Hence, it is now reasonable to consider the application of a genetic algorithm for optimizing the selection of these parameters for dynamic coordination of the goals, i.e.,

$$
Q_{1}=f_{1}\left(\rho_{m}, \rho_{M}\right) ; \quad W_{2}=f_{2}\left(\rho_{m}, \rho_{M}\right)
$$

The actual problem is that unwanted situations may appear, if the genetic algorithm search is limited by the $\rho_{m}$ and $\rho_{M}$, which specify the near and far concepts in executing the two primary parking rules. Particularly, when the vehicle entered the respective area of coefficient $W_{2}$ (that is, when the vehicle is close to the target), it will park parallel to the space, but it will not park inside it if it reached this area through "connection" with the straight line determined by the prolongation of the space direction.

Thus, the genetic algorithm would have the responsibility of optimization of $\Delta_{1}$ and $\Delta_{2}$, in addition to $\rho_{m}$ and $\rho_{M}$.

A fuzzy like meta-heuristic performs global coordination, which is optimized by a genetic algorithm. Let again consider how a human driver parks, it is found that one highly efficient maneuver, if no obstacles are faced, is approaching, in almost any direction, a region near to the direction and position of space. Then, priority is assigned to heading, though not absolute. When the vehicle is in line with the space, the approach goal gives the highest priority, albeit again not an exclusive priority. We want to qualitatively examine the implementation of this maneuver.

Step 1. Transfer area approach. This step can be executed with no concern about the heading goal J2. Nevertheless, the vehicle's position in this transfer area needs to be as aligned as possible regarding the space direction.

Step 2. Aligning with the space direction. When its position is in the transfer area or sub-target area, the vehicle assigns the highest priority to the heading goal, albeit not an exclusive priority. Certainly, the more alignment it has, the easier the maneuver is.

Step 3. Parking space approach in accordance with the respective heading. Following aligning with the space direction, the mere concern of the vehicle would be the reduction of its distance to space 
(highest priority of index $\mathrm{J} 1$ ). Goal $\mathrm{J} 2$ should gain some influence, though a very weak so that probable loss of alignment is prevented.

\subsection{Phase 3: Cuckoo search algorithm to increase driver information from the surroundings}

In this paper, the proposed algorithm is on a crowd-based method. Optimal utilization of space, time, and cost is undoubtedly one of the most important principles in different decision-makings. In a smart car park equipped with smart guidance system, every part of the parking is equipped with a car presence or absence detector.

These detectors come in different ultrasonic, magnetic, and camera sensor varieties which with their unique features, they are suitable for indoor and outdoor car parks. Also, some of the models have integrated detectors and indicators. Indicators are in charge of notifications regarding each parking spot being full/empty. To be informed of empty parking spots, it suffices for the driver to pay attention to the indicators installed in each parking spot when entering each corridor. With various green/red/yellow/blue colors, indicators show the empty/full/reserved/handicapped parking spots. The indicator panel shows the number of empty spaces in different parts of the parking. Urban empty space indicator panels work wirelessly and by installing them in key urban points leading to car parks, they declare the empty spaces in car parks. The control software is a comprehensive system software capable of displaying the complete 2D plan of the parking and specifying full and empty locations. Among the special features of this software is the capability of providing hourly, daily, weekly, and monthly statistics.

Using control systems and giving an RFID card to each car park user, in addition to registering an ID for each car, the ability to be informed of exact parking location, entrance and departure time, parking usage recurrence, and other items can be provided. Furthermore, some parking spots could be considered as reserved for parking's regular customers or VIP ones. This way, special cars would no longer need to get a car park entrance card and when returning to the parking, the barrier automatically goes up for them. This system is considered a small part of smart parking management and guidance system. The capabilities of this option include recording all of car's information, making a backup of special information, ability to provide service to the users, and the ability to consider parkway for special spots where by entering them, barrier is removed automatically.

Also, NFC control systems are one of the novel technologies used in smart parking systems. In the communication system using this system, drivers can easily pay their fee with their smartphone when leaving, without the need for cash. Two of the capabilities of this system are paying the parking fee without the need for cash and controlling driver's coming and going using a smart card. Using smart optimization algorithms, management in smart car parks can be taken with the approach to increase the parking efficiency. Using heuristic methods, a template can be presented that while maximizing profits for the parking owner, it guarantees decreased time and space expenses of car owners. The proposed template will also follow the policy during low load hours and injection into the network during high load hours.

In the proposed parking management template, planning will be done separately for each and every car. Also, each car is notified of its expenses at the time of entrance and based on the reduction in expenses, it will have a better view on selecting or not selecting. The smart optimization algorithm used in this study for finding the optimal cars' proposed template is the Cuckoo optimization 
algorithm, which is one of the best smart algorithms with high convergence speed and super easy implementation. In this regard, finding sports suitable for parking a car in busy cities, whether in main streets and their adjacent streets or in existing parking building, is very difficult. With the help of proposed management templates, urban users will have the possibility to receive smart parking spot information in adjacent streets using the applications on their phone or the navigation system of the car in real time. Finding a spot for parking the car is very painful most of the time. The thing that leads to this is that you will reach your intended location which does not have a location suitable for parking the car and even in the best scenario, by consuming indefinite amount of time and fuel, you will park your car. According to the latest international transportation management studies, finding a suitable location for parking takes around 10 minutes in a developed country like Germany while in Italy, it takes 15 minutes, which includes navigating something around 4.5 kilometers from adjacent street which will lead to the unnecessary emission of 1.3 kilograms of carbon dioxide gas created by the car into the atmosphere. The studies have also shown that about 30 percent of drivers in dense urban areas are looking for a spot suitable for parking. An interesting interpretation of all the trips taken in a small urban area by these drivers is that it is equal to 14 trips on all the roads on planet earth.

Using the proposed template, management and satisfaction of constraints in different positions is considered and urban users will be notified of location spots available between the origin and destination of their trip without any effort or cost. Finding a suitable parking spot using a Radar Eye in a smart car park is shown in Figure 4.

\section{Parking Management}

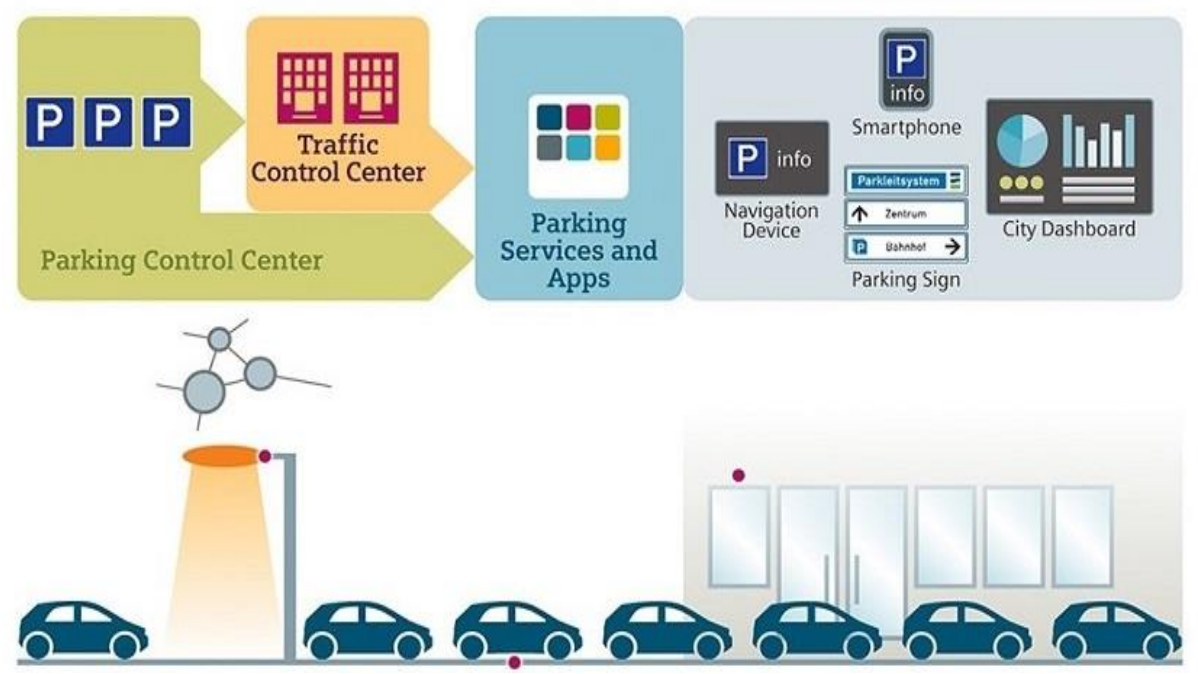

Fig. 4 Finding a suitable parking spot using a Radar Eye in a smart car park.

The self-trained operation of the smart parking system is such that sensor outputs are sent to the control system using the cellular network. Once there, they are analyzed such that the state of available parking spots is identified and suitable information for improving the situation of finding parking space is sent to the related application and cars' navigation systems through the internet.

In the control center, analysis of the obtained data will be carried out using the Cuckoo algorithm to optimize the problem. The proposed approach is on the self-trained operation of the system software. The scheduling software analyzes parking spots getting empty in crowded locations and based on statistic and real-time information, it sends correct predictions for future times to the drivers. 
Also, the system helps the drivers in choosing their form of transportation such that before getting in the car and starting their trip to their destination, it sends some information to the driver. Based on this information, in case of crowdedness and lack of enough parking space in the streets adjacent to the driving destination, the driver is prohibited from driving with private car and the application proposes the use of public transportation facilities.

The smart parking management system is a modular sensor ultrastructure where the connection of sensors to the control center is enabled with the help of the internet of things (IoT) platform. In fact, the intended structure is one of the bases for forming the concept of a smart city. In case the RFID system is added to any of the existing parking spaces, it is possible to add validator devices as well, which as an example, enabling the users of specific locations to use the private parking space devoted to that location or calculating parking duration and offering the parking ticket will be some of the features of this system. The Siemens parking management solution has taken some steps beyond the aforementioned items and has presented systems like urban traffic management, adaptive lighting management, information analysis system for urban areas, and public urban services. The result and overall assessment leads to having a "smart and innovative city" with smart strategic management.

\section{Evaluating the Performance}

The automatic parking performance is assessed in the following section.

\subsection{Performance metrics}

Here, the performance and effectiveness of our suggested MODM-Based solution are systematically assessed with complete simulations.

FPR: The FP is calculated by vehicles that parked correctly but were mistakenly identified as the vehicles that parked incorrectly. [25-32]. Therefore, the is defined as illustrated in Eq. (12).

$$
F P R=\left(\frac{F P R}{F P R+T N R}\right) * 100 \quad \text { Where: } \quad T N R=\left(\frac{T N R}{T N R+F P R}\right) * 100
$$

FNR: FP is calculated by vehicles that are parked incorrectly but are mistakenly identified as vehicles that parked correctly [33-38]. Eq. (13) demonstrates the calculation.

$$
F N R=\left(\frac{T P R+T N R}{A l l}\right) * 100 \quad \text { Where: } \quad T P R=\left(\frac{T P R}{T P R+F N R}\right) * 100
$$

DR: Ratio of the number of vehicles parked correctly divided by the total number of vehicles parked. DR is calculated by Eq. (14) [39-42].

$$
D R=\left(\frac{T P R}{T P R+F N R}\right) * 100 \quad \text { where } \quad A l l=T P R+T N R+F P R+F N R
$$




\subsection{Simulation results}

In this section, we analyze the performance of MODM-Based solution. The difficulties in implementation and debugging facility location-allocation problem in real networks, raises the necessity to consider simulations as a fundamental design tool. The main advantage of simulation is simplifying analysis and protocol verification, mainly in large-scale systems. It is possible to employ a NAM in the Network Simulator (NS-3) to visualize the results. In this section, the performance of our proposed approach is evaluated using NS-3 on Linux Ubuntu 18.04 LTS as the simulation tool, and the results are discussed further. Table 6-8 compares the performance of MODM-Based solution vs CSA method [42] in terms of FPR, FNR, and DR.

Table 6: FNR (in \%) (With MODM-Based solution vs CSA Method)

\begin{tabular}{|c|c|c|}
\hline Vehicles & $\begin{array}{c}\text { With MODM-Based } \\
\text { solution }\end{array}$ & CSA Method \\
\hline 50 & 16.4 & 33.8 \\
\hline 100 & 16.7 & 34.5 \\
\hline 150 & 16.8 & 35.2 \\
\hline 200 & 17.1 & 35.9 \\
\hline 250 & 17.3 & 36.4 \\
\hline 300 & 17.4 & 37.3 \\
\hline 350 & 17.5 & 38.2 \\
\hline 400 & 17.8 & 39.3 \\
\hline 450 & 18.4 & 40.5 \\
\hline 500 & 18.8 & 41.7 \\
\hline
\end{tabular}

Table 7: FPR (in \%) (With MODM-Based solution vs CSA Method)

\begin{tabular}{|c|c|c|}
\hline Vehicles & $\begin{array}{c}\text { With MODM-Based } \\
\text { solution }\end{array}$ & CSA Method \\
\hline 50 & 27.3 & 12.3 \\
\hline 100 & 27.9 & 12.5 \\
\hline 150 & 28.6 & 12.7 \\
\hline 200 & 29.5 & 12.9 \\
\hline 250 & 30.1 & 13.3 \\
\hline 300 & 31.4 & 13.8 \\
\hline 350 & 31.9 & 14.3 \\
\hline 400 & 32.5 & 14.7 \\
\hline 450 & 32.8 & 14.8 \\
\hline 500 & 33.6 & 15.4 \\
\hline
\end{tabular}

Table 8: DR (in \%) (With MODM-Based solution vs CSA Method)

\begin{tabular}{|c|c|c|}
\hline Vehicles & $\begin{array}{c}\text { With MODM-Based } \\
\text { solution }\end{array}$ & CSA Method \\
\hline 50 & 58.6 & 87.4 \\
\hline 100 & 58.9 & 88.5 \\
\hline 150 & 59.3 & 89.3 \\
\hline
\end{tabular}




\begin{tabular}{|l|l|l|}
\hline 200 & 59.7 & 90.2 \\
\hline 250 & 60.1 & 90.8 \\
\hline 300 & 60.7 & 91.5 \\
\hline 350 & 61.2 & 91.7 \\
\hline 400 & 61.9 & 92.4 \\
\hline 450 & 62.3 & 92.9 \\
\hline 500 & 62.9 & 93.5 \\
\hline
\end{tabular}

FNR: Figure 6 shows the comparison between the MODM-Based solution vs CSA Method. The proposed method performs better in the number of cars from 50 to 500 than the CSA Method. Due to the increasing number of cars and road traffic, the amount of car park space has also decreased. The main reason is the information error. The driver cannot respond appropriately because he does not receive or receive the necessary information late. With the help of the cuckoo search algorithm and considering the limitations, it extends the level of driver information from the surrounding environment, and is facilitated by the exchange of information using an app that delivers information to the driver much faster and the driver responds at the right time. Update. As a result, the MODM-Based solution has a significant improvement over the CSA Method. As demonstrated in the Fig. 5, WSN-FAHN increases the FNR by more than $30 \%$ those of CSA Method.

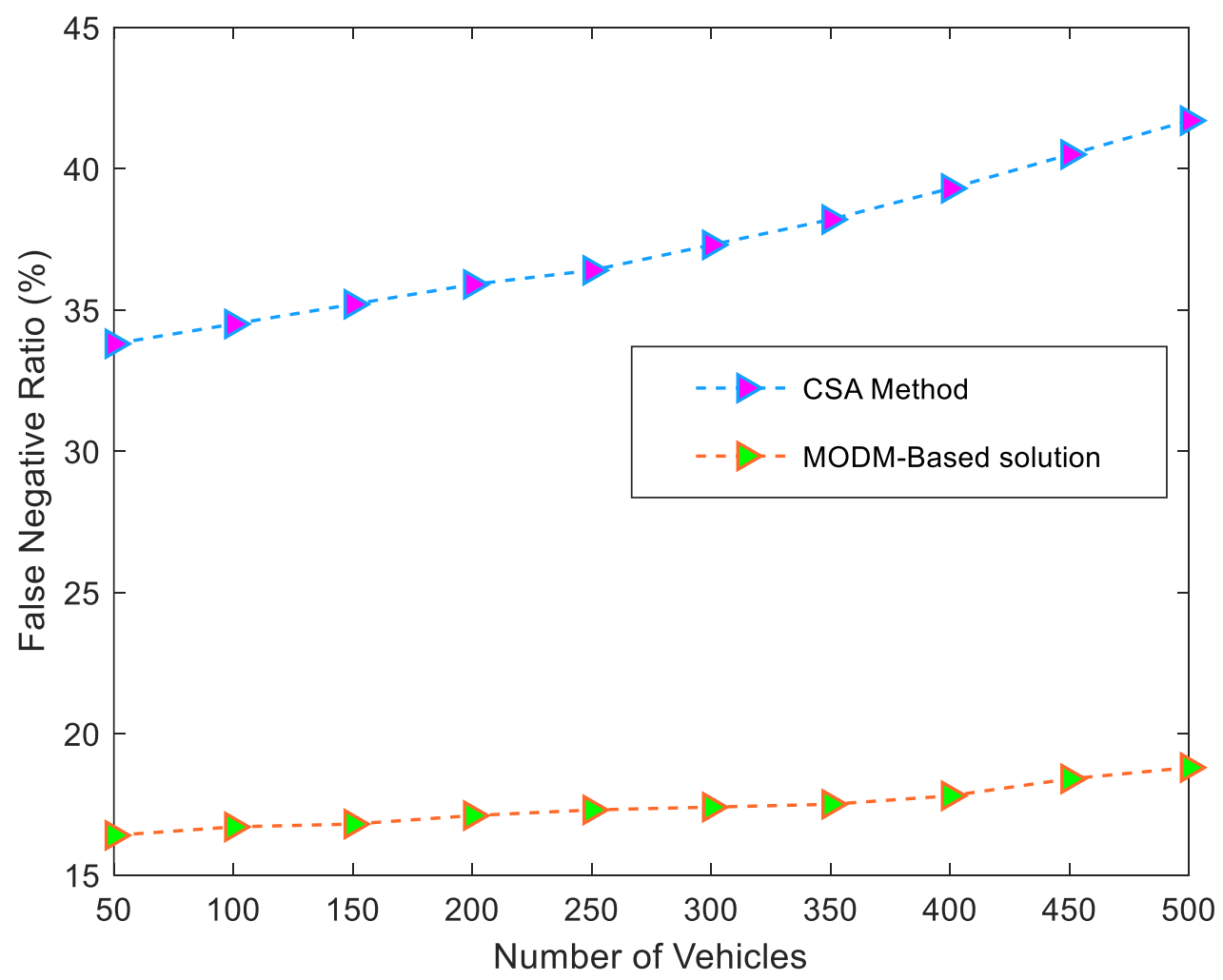

Fig. 5 Comparison of the MODM-Based solution vs CSA Method in term of FNR. 
FPR: Figure 6 shows the comparison between the MODM-Based solution vs CSA Method in term of FPR. As shown in Fig. 6, the proposed method performs better than the CSA Method because the predominance of the proposed method is that in the parking management, individual planning will be performed for each vehicle. And each car will be informed of its cost at the time of entry and will have a better view of whether or not to choose based on the reduced cost. The intelligent optimization algorithm used in this study is to find the customer optimization algorithm for cuckoo optimization. Which is one of the most suitable intelligent algorithms with high convergence speed and very easy implementation. In this regard, it is very difficult to find the right car park space in crowded cities, whether on the main streets and adjacent streets or in existing parking structures. With the proposed management templates, urban users will be able to instantly access smart parking spaces available on the adjacent streets through mobile app or car navigation system for an instant. As demonstrated in the Fig. 6, WSN-FAHN increases the DR by more than $29 \%$ those of CSA Method.

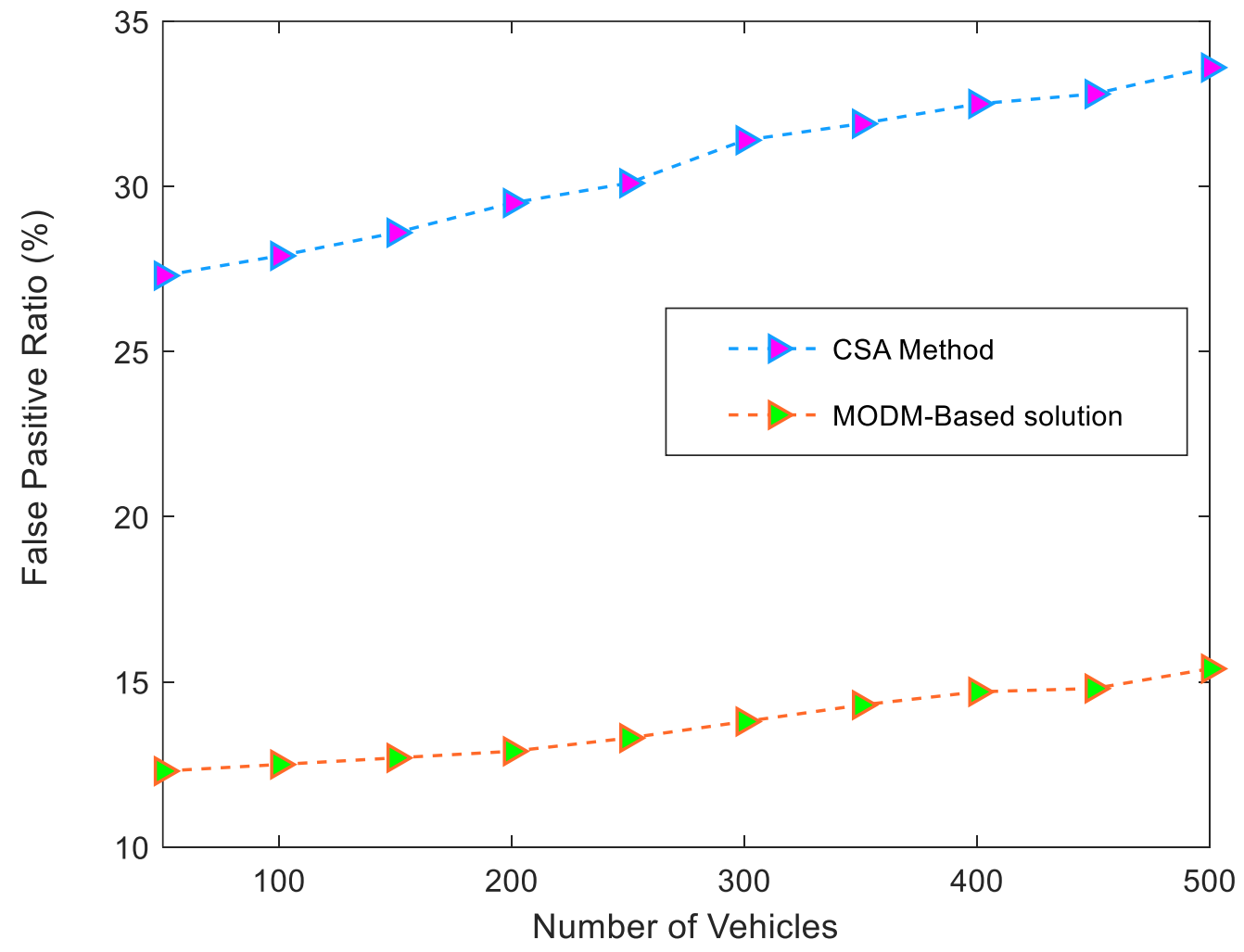

Fig. 6 Comparison of the MODM-Based solution vs CSA Method in term of FPR.

DR: Figure 7 shows the comparison between the MODM-Based solution vs CSA Method in term of DR. As shown in Figure 8, the proposed method performs better than the CSA Method because the superiority of the proposed method is that management and satisfaction constraints are considered in different 
situations and urban users without any effort and cost of information. Parks will be notified between the source and destination of their trip. As demonstrated in the Fig. 7, WSN-FAHN increases the DR by more than $33 \%$ those of CSA Method.

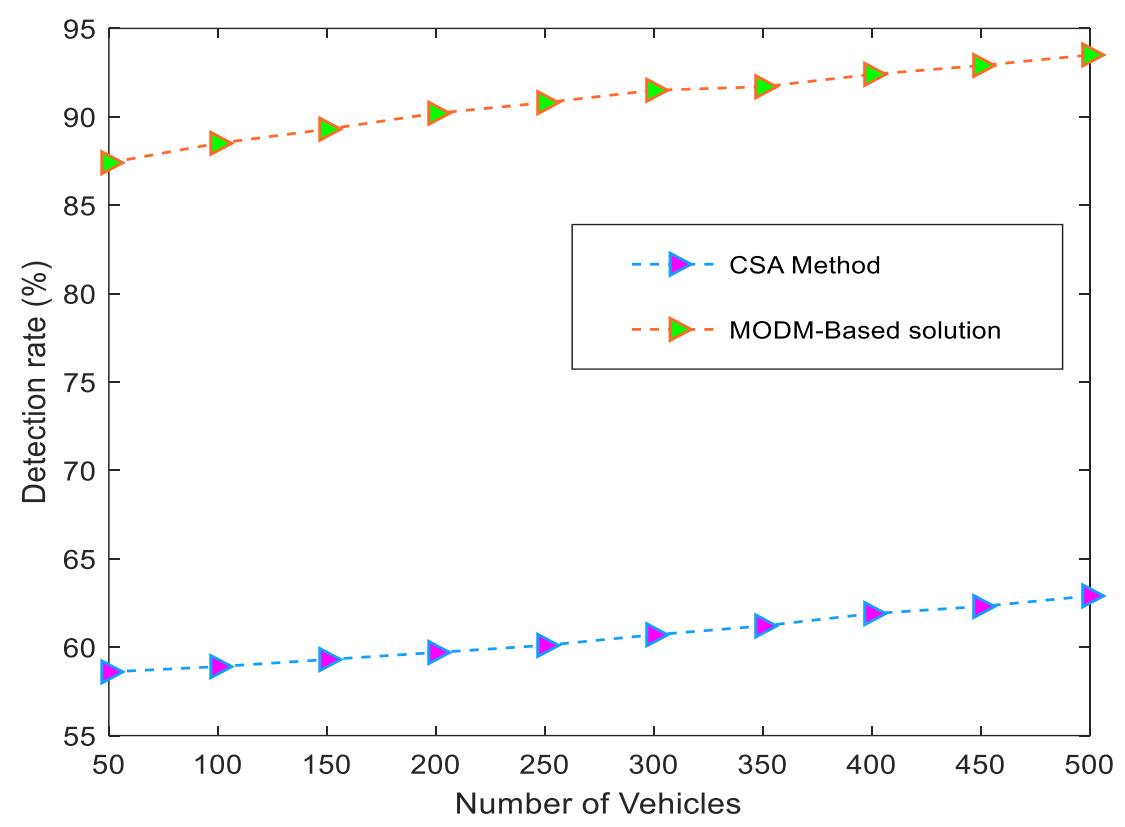

Fig. 7 Comparison of the MODM-Based solution vs CSA Method in term of DR.

\section{Conclusion}

This paper uses two phases: the first phase, for goal coordination, was used genetic algorithms and the Cuckoo search algorithm was used to increase driver information from the surroundings. Using the Cuckoo search algorithm and considering the limitations, it increases the driver's level of information from the environment. Also, by exchanging information through the application, it enables the information to reach the driver much more quickly and the driver reacts appropriately at the right time. Finally, we have also considered a more complex situation in which a priori unknown obstacles are present in the parking place. By adding a new objective (i.e., collision avoidance) to the multi-criteria performance function the vehicle is able to park without colliding with the existing obstacles. The results confirmed that our scheme is capable of exhibiting high prediction accuracy (exceeding 90.93\%). It addition, our proposed scheme has high speed (more than 76.41\%), low FPR (less than 4.29\%), and low FNR (less than 5.15\%), in comparison with the other approaches currently being employed.

\section{Conflict of Interest}

None.

\section{Reference}

1. Montemerlo M, Thrun S, Dahlkamp H, Stavens D, Strohband S. Winning the DARPA Grand Challenge with an AI robot. InAAAI 2006 Jul 16 (pp. 982-987).

2. Fotohi, R., \& Effatparvar, M. (2013). A cluster based job scheduling algorithm for grid computing. International Journal of Information Technology and Computer Science (IJITCS), 5(12), 70-77. 
3. Binder A, Montavon G, Lapuschkin S, Müller KR, Samek W. Layer-wise relevance propagation for neural networks with local renormalization layers. InInternational Conference on Artificial Neural Networks 2016 Sep 6 (pp. 63-71). Springer, Cham.

4. Arras L, Horn F, Montavon G, Müller KR, Samek W. Explaining predictions of non-linear classifiers in NLP. arXiv preprint arXiv:1606.07298. 2016 Jun 23.

5. Fotohi, R., Ebazadeh, Y., \& Geshlag, M. S. (2016). A new approach for improvement security against DoS attacks in vehicular ad-hoc network. International Journal of Advanced Computer Science and Applications, 7(7), 10-16.

6. Thrun S, Montemerlo M, Dahlkamp H, Stavens D, Aron A, Diebel J, Fong P, Gale J, Halpenny M, Hoffmann G, Lau K. Stanley: The robot that won the DARPA Grand Challenge. Journal of field Robotics. 2006 Sep;23(9):661-92.

7. Hatt M, Parmar C, Qi J, El Naqa I. Machine (deep) learning methods for image processing and radiomics. IEEE Transactions on Radiation and Plasma Medical Sciences. 2019 Mar 1;3(2):104-8.

8. Kocić J, Jovičić N, Drndarević V. An End-to-End Deep Neural Network for Autonomous Driving Designed for Embedded Automotive Platforms. Sensors. 2019 Jan;19(9):2064.

9. Nawrocka D. Machine learning for trading and portfolio management using Python.

10. Henderson P, Islam R, Bachman P, Pineau J, Precup D, Meger D. Deep reinforcement learning that matters. InThirtySecond AAAI Conference on Artificial Intelligence 2018 Apr 29.

11. Fotohi, R., \& Jamali, S. (2014). A comprehensive study on defence against wormhole attack methods in mobile Ad hoc networks. International journal of Computer Science \& Network Solutions, 2, 37-56.

12. Xie Y, Le L, inventors; Equifax Inc, assignee. Dual deep learning architecture for machine-learning systems. United States patent application US 16/141,152. 2019 Mar 28.

13. Kanade T, Thorpe C, Whittaker W. Autonomous land vehicle project at CMU. InProceedings of the 1986 ACM fourteenth annual conference on Computer science 1986 Feb 1 (pp. 71-80). ACM.

14. Fotohi, R., Jamali, S., Sarkohaki, F., \& Behzad, S. (2013). An Improvement over AODV routing protocol by limiting visited hop count. International Journal of Information Technology and Computer Science (IJITCS), 5(9), 87-93.

15. Fotohi, R., Jamali, S., \& Sarkohaki, F. (2013). Performance Evaluation of AODV, LHC-AODV, OLSR, UL-OLSR, DSDV Routing Protocols. International Journal of Information Technology and Computer Science (IJITCS), 5, 21.

16. Wallace RS, Stentz A, Thorpe CE, Moravec HP, Whittaker W, Kanade T. First Results in Robot Road-Following. InIJCAI 1985 Aug 18 (pp. 1089-1095).

17. Zong, F., Yu, P., Tang, J., \& Sun, X. (2019). Understanding parking decisions with structural equation modeling. Physica A: Statistical Mechanics and its Applications, 523, 408-417.

18. Zong, F., Yu, P., Tang, J., \& Sun, X. (2019). Understanding parking decisions with structural equation modeling. Physica A: Statistical Mechanics and its Applications, 523, 408-417.

19. Fotohi, R., \& Bari, S. F. (2020). A novel countermeasure technique to protect WSN against denial-of-sleep attacks using firefly and Hopfield neural network (HNN) algorithms. The Journal of Supercomputing, 1-27.

20. Fotohi, R. (2020). Securing of Unmanned Aerial Systems (UAS) against security threats using human immune system. Reliability Engineering \& System Safety, 193, 106675.

21. Maravall, D., \& de Lope, J. (2007). Multi-objective dynamic optimization with genetic algorithms for automatic parking. Soft Computing, 11(3), 249-257.

22. Gao, G., Sun, H., \& Wu, J. (2019). Activity-based trip chaining behavior analysis in the network under the parking fee scheme. Transportation, 46(3), 647-669.

23. Korhonen, Juha, and Tatu Salminen. "Method and system for providing information based on location of userdevice." U.S. Patent Application 10/182,312, filed January 15, 2019

24. Fotohi, R., Firoozi Bari, S., \& Yusefi, M. (2020). Securing wireless sensor networks against denial-of-sleep attacks using RSA cryptography algorithm and interlock protocol. International Journal of Communication Systems, 33(4), e4234.

25. Zhang, L., Wang, Y. P., Sun, J., \& Yu, B. (2019). The sightseeing bus schedule optimization under park and ride system in tourist attractions. Annals of Operations Research, 273(1-2), 587-605.

26. Zhang, C., Wang, Q., Wang, M., Chen, J., Liu, H., \& Fu, S. (2018, August). Intelligent Parking Management System Design from a Mobile Edge Computing (MEC) Perspective. In 2018 IEEE 88th Vehicular Technology Conference (VTC-Fall) (pp. 1-5). IEEE.

27. Ornstein, J., Ornstein, J., \& Rathod, N. (2019). U.S. Patent Application No. 16/142,113.

28. Segwick, T. (2002). THE KEY ROLE OF PARKING IN AN INTELLIGENT TRANSPORT INDUSTRY. In 9th World Congress on Intelligent Transport SystemsITS America, ITS Japan, ERTICO (Intelligent Transport Systems and Services-Europe). 
29. Lan, K. C., \& Shih, W. Y. (2014). An intelligent driver location system for smart parking. Expert Systems with Applications, 41(5), 2443-2456.

30. Marjani, S. R., Gheibi, M., Talavat, V., \& Farsadi, M. (2015, September). A novel hybrid intelligent method for static var compensator placement in distribution network with plug-in hybrid electrical vehicles parking. In 2015 Intl Aegean Conference on Electrical Machines \& Power Electronics (ACEMP), 2015 Intl Conference on Optimization of Electrical \& Electronic Equipment (OPTIM) \& 2015 Intl Symposium on Advanced Electromechanical Motion Systems (ELECTROMOTION) (pp. 323-330). IEEE.

31. Fotohi, R., Heydari, R., \& Jamali, S. (2016). A Hybrid routing method for mobile ad-hoc networks. Journal of Advances in Computer Research, 7(3), 93-103.

32. Maravall, D., \& de Lope, J. (2007). Multi-objective dynamic optimization with genetic algorithms for automatic parking. Soft Computing, 11(3), 249-257.

33. Babhulkar A. Self-driving car using udacity's car simulator environment and trained by deep neural networks.

34. Zaminkar, M., Sarkohaki, F., \& Fotohi, R. A method based on encryption and node rating for securing the RPL protocol communications in the IoT ecosystem. International Journal of Communication Systems, e4693.

35. Faraji-Biregani, M., \& Fotohi, R. (2020). Secure communication between UAVs using a method based on smart agents in unmanned aerial vehicles. The Journal of Supercomputing, 1-28.

36. Fotohi, R., Nazemi, E., \& Aliee, F. S. (2020). An Agent-Based Self-Protective Method to Secure Communication between UAVs in Unmanned Aerial Vehicle Networks. Vehicular Communications, 100267.

37. Zaminkar, M., \& Fotohi, R. (2020). SoS-RPL: Securing Internet of Things Against Sinkhole Attack Using RPL Protocol-Based Node Rating and Ranking Mechanism. WIRELESS PERSONAL COMMUNICATIONS.

38. Sarkohaki, F., Fotohi, R., \& Ashrafian, V. (2020). An efficient routing protocol in mobile ad-hoc networks by using artificial immune system. arXiv preprint arXiv:2003.00869.

39. Mabodi, K., Yusefi, M., Zandiyan, S., Irankhah, L., \& Fotohi, R. (2020). Multi-level trust-based intelligence schema for securing of internet of things (IoT) against security threats using cryptographic authentication. The Journal of Supercomputing, 1-26.

40. Seyedi, B., \& Fotohi, R. (2020). NIASHPT: a novel intelligent agent-based strategy using hello packet table (HPT) function for trust Internet of Things. The Journal of Supercomputing, 1-24.

41. Jamali, S., Fotohi, R., \& Analoui, M. (2018). An artificial immune system based method for defense against wormhole attack in mobile adhoc networks. Tabriz Journal of Electrical Engineering, 47(4), 1407-1419.

42. Jamali, S., \& Fotohi, R. (2017). DAWA: Defending against wormhole attack in MANETs by using fuzzy logic and artificial immune system. the Journal of Supercomputing, 73(12), 5173-5196.

43. Jamali, S., \& Fotohi, R. (2016). Defending against wormhole attack in MANET using an artificial immune system. New Review of Information Networking, 21(2), 79-100.

44. Yang, Z., \& Ma, D. (2002). Study on the implement technologies of urban traffic flow guidance system. In Traffic And Transportation Studies (2002) (pp. 690-695). 